\title{
ESCALA DE CARGA DEL CUIDADOR DE ZARIT: EVIDENCIA DE VALIDEZ EN MÉXICO
}

\author{
ZARIT CAREGIVER BURDEN SCALE: EVIDENCE OF VALIDITY IN MEXICO
}

Xolyanetzin Montero Pardo', Samuel Jurado Cárdenas', Alejandra Valencia Cruz', José Méndez Venegas' e lgnacio Mora Magaña ${ }^{2}$

'Universidad Nacional Autónoma de México, México, D. F., México

${ }^{2}$ Instituto Nacional de Pediatría, México, D. F., México

Resumen

El propósito fue obtener evidencia de validez de la escala de carga del cuidador de Zarit en cuidadores primarios informales de niños con alguna enfermedad crónico degenerativa, en un hospital de la ciudad de México. La muestra constó de 252 participantes. Se utilizó la escala de carga del cuidador de Zarit (CBI), el cuestionario general de salud (GHQ-28) y el cuestionario de estrés percibido (PSS). El análisis factorial exploratorio y confirmatorio de la escala mostró que está conformada por tres factores que explican el $50 \%$ de la varianza. Tuvo un alfa de Cronbach de $\mathbf{0 , 8 4}$. y el modelo tuvo un buen ajuste con valores iguales o superiores a 0,90. La validez de criterio entre el GHQ-28 y el CBI arrojó un valor $r=0,46$. La validez de constructo se confirmó correlacionando el PSS y la CBI obteniendo un valor $r=0,36$.

Palabras clave: Impacto del cuidado, calidad de vida, propiedades psicométricas, análisis factorial confirmatorio.
Abstract

The purpose was to obtain evidence of the validity of the Zarit Burden Scale in informal primary caregivers of children with chronic illness, in a hospital in Mexico City. The sample consisted of 252 participants. The instruments used were Zarit Burden Interview (CBI), General Health Questionnaire (GHQ28) and the Perceived Stress Scale (PSS). The exploratory and confirmatory factor analysis of the scale resulted in three factors which explain $50 \%$ of the variance. Cronbach's alpha was .84 and the model fit well with values equal to or above 0.90 . The criterion validity obtained between the GHQ-28 and the CBI, showed a value $r=0.46$. The construct validity was estimated correlating the PSS and the CBI, obtaining a value $r=0.36$.

Key Words: Impact of care, quality of life, psychometric properties, confirmatory factor analysis.

\section{INTRODUCCIÓN}

Los avances científicos en medicina aumentan la expectativa de vida al controlar una serie de enfermedades que antes conducían a una muerte inminente; esto ocasiona que un número considerable de pacientes en algún momento de su vida requieran de personas que se hagan cargo de su cuidado y atención por un periodo no determinado; a esta persona se le llama "cuidador" (1).

\section{Correspondencia:}


Las enfermedades que conducen a la necesidad de recibir cuidados son variadas y se encuentran principalmente en los extremos de la vida. En la vejez como efecto del deterioro psíquico o enfermedades físicas y en la niñez por problemas crónicos como asma, epilepsia, cáncer, diabetes o discapacidades $^{(2)}$.

Dentro del sistema de salud se han identificado dos tipos de cuidadores: los formales ubicados dentro del Sistema Institucional y los llamados informales insertados en el Sistema Doméstico de Cuidado de la Salud ${ }^{(3)}$. El cuidador informal se define a partir de dos características: 1) "no es remunerado" lo cual le hace parecer con demasiada frecuencia como un tipo de cuidado "no válido" y 2) se desarrolla en el ámbito de las relaciones familiares ${ }^{(4,5)}$.

Los cuidadores informales, a su vez, se dividen en primarios y secundarios, la diferencia entre estos es el grado de responsabilidades que asumen hacia el paciente y el tiempo que dedican a cuidar al mismo; en este contexto, el cuidador primario es el encargado de tomar tanto la mayoría de las decisiones como invertir su tiempo acompañando al enfermo, mientras el secundario sólo es el relevo. Así mismo, organizan su vida en función del enfermo, y los cuidados proporcionados tienen que hacerse compatibles con las actividades de la vida cotidiana(6).

El cuidador debe hacer frente a las demandas del cuidado y a su vez asumir el rol familiar que siempre llevó a cabo antes de la enfermedad de su paciente. A partir de las demandas propias del rol puede presentar síntomas físicos, psicológicos, conductuales y sociales que ocasionan carga, como consecuencia de esto disminuye la calidad de la atención hacia el enfermo y sus relaciones sociales, familiares y conyugales ${ }^{(7)}$.

Para Zarit, Reever y Bach-Peterson(11), la carga del cuidador hace referencia a las actitudes y reacciones emocionales ante la experiencia de cuidar y al grado de perturbaciones o cambios en diversos aspectos del ámbito doméstico y de la vida de los cuidadores. La carga es una valoración o interpretación que hace el cuidador ante los estresores derivados del cuidado en función de los recursos y habilidades con lo que éste cuenta ${ }^{(8)}$. La carga interfiere en el manejo adecuado del enfermo y en la propia evolución de la enfermedad, provocando al mismo tiempo el deterioro de la calidad de vida del cuidador ${ }^{(6)}$.

La carga es un concepto clave en la investigación de las repercusiones del cuidado de los pacientes crónicos sobre la familia y el cuidador. El término se remonta a los años 60, del siglo pasado, cuando Grad y Sainsbury ${ }^{(9)}$ realizaron una investigación para conocer qué efectos tenía sobre la vida cotidiana de las familias el hecho de tener a un miembro con una enfermedad psiquiátrica. Para ello evaluaron la carga económica y emocional ocasionada por tener este tipo de enfermos. En los años 80 se realizaron estudios acerca de las consecuencias del cuidado de ancianos con síndromes demenciales, permitiendo establecer las repercusiones negativas que el rol tenía para los cuidadores. Lo anterior, se trasladó al estudio de cuidadores de adultos con diversos tipos de enfermedades crónicas ${ }^{(10)}$.

Zarit y su grupo de colaboradores consideraron la carga de los cuidadores como la clave para estudiar el desgaste ocasionado por el cuidado de personas con demencia $^{(11)}$ y las investigaciones relacionadas con carga han dado origen a diversos instrumentos de medición que tomaron en cuenta dimensiones como la carga objetiva, subjetiva y evaluaciones globales de la misma $^{(8)}$, siendo hasta el momento, el más utilizado la escala de carga del cuidador de Zarit. Dicho instrumento contaba, originalmente, con 29 ítems diseñados para evaluar la carga subjetiva asociada al cuidado de ancianos, teniendo en cuenta los 
siguientes aspectos: salud física y psicológica, área económica y laboral, relaciones sociales y relación con la persona receptora de cuidados. En su revisión se redujo el número de ítems a $22^{(12)}$.

La entrevista de carga del cuidador de Zarit ha sido traducida y validada en diferentes países con adultos mayores dependientes, adultos con enfermedades crónicas degenerativas y/o enfermedades mentales. El número de factores que se han encontrado oscila entre tres y cinco, aunque es importante señalar que no hay un acuerdo respecto a cómo se deben Ilamar esos factores, por ejemplo: impacto del cuidado vs sobrecarga, relación interpersonal vs de dependencia, competencia vs autoeficacia ${ }^{(8,10,13-17)}$.

Así mismo, los trabajos respecto a la carga de cuidadores de niños con enfermedades crónicas son escasos, en comparación con la cantidad de estudios centrados en los cuidadores de adultos. La literatura científica muestra que hay evidencia de que para evaluar la carga de los cuidadores de niños con enfermedades crónicas, usualmente se emplea la escala original de Zarit desarrollada para adultos mayores dependientes ${ }^{(18-20)}$, la validación realizada para cuidadores de adultos con enfermedades crónicas $^{(21-25)}$ u otros instrumentos elaborados para estudios específicos ${ }^{(26)}$.

Es decir, no se ha realizado una validación de la escala específica para cuidadores de niños con enfermedades crónicas a pesar de que la estructura factorial de la misma puede variar al ser aplicada a una muestra, donde a pesar de que vive una situación similar a la de los cuidadores de adultos respecto a la presencia de una enfermedad crónica, los antecedentes del cuidado $^{(27)}$; es decir, la historia de la relación establecida previa a la aparición de la enfermedad entre el cuidador y la persona a la que cuida.

Esta se establece en un contexto distinto dependiendo de la edad de la persona a la que cuidan, tanto por los roles implicados como por las características afectivas y emocionales de la relación, ya que en cuidadores de adultos mayores es un relación principalmente entre padre/madrehija ${ }^{(28,29,39)}$, mientras que en cuidadores de niños es entre madre-hijo ${ }^{(31-36)}$.

La hipótesis del estudio se deriva de que la relación entre el cuidador y la persona a la que cuida puede influir en cómo percibe la situación de cuidado y el impacto que este tiene en su vida. Ya que esto es distinto dependiendo de la edad de la persona receptora de cuidados; mientras los cuidadores de adultos mayores asumen este rol como "una obligación moral" basada en la historia de la relación establecida previamente entre cuidador y persona cuidada en función de si esta fue cercana o conflictiva y como agradecimiento por el apoyo y amor que el adulto mayor brindo durante su vida al cuidador; sin embargo, con el impacto que el cuidado tiene en diversas áreas de la vida del cuidado, aparecen en este sentimientos conflictivos y ambivalentes respecto a si debían ser los cuidadores y continuar con la ejecución de su rol o cederlo a otros familiares (30); las madres cuidan a sus hijos como una extensión de sus deberes domésticos, considerando el cuidado como un "deber natural, que nadie está a la altura de cumplir tal como ellas lo hacen"(20,37); a partir de esta creencia establecen un fuerte vínculo emocional con sus hijos y una abnegación maternal que coloca las necesidades de estos como lo más importante en la vida de la mujer ${ }^{(38)}$.

Por lo antes mencionado, las creencias transmitidas culturalmente sobre la maternidad influyen en el desempeño del rol de cuidador que la madre ejerce, y es a partir de estas creencias que aunque la mujer perciba que diversas áreas de su vida se han impactado por el cuidado continuara con el desempeño del rol, porque lo más importante para ella no es su salud físi- 
ca y/o mental sino el bienestar del hijo ${ }^{(38)}$. Mientras que las cuidadoras de adultos al percibir el impacto del cuidado en diversas áreas de su vida empiezan a surgir emociones ambivalentes respecto a continuar con el rol de cuidador que impacta la ejecución del mismo por tener conductas de maltrato por acción u omisión hacia la persona que cuida(28-30).

A partir de lo antes descrito, el problema que se pretendió abordar en este estudio fue la falta de un instrumento válido y confiable para evaluar la carga del cuidador de niños con enfermedades crónicas que tome en cuenta las características específicas de la relación en la que se desarrolla el cuidado, como un primer paso para desarrollar un modelo de atención psicológica para atender a esta población.

En esta dirección, el propósito del presente trabajo, fue analizar el comportamiento psicométrico de la escala de carga del cuidador de Zarit en una población de cuidadores primarios informales de niños con enfermedades crónico degenerativas que reciben atención médica en un hospital de tercer nivel en la ciudad de México. Así como, determinar su validez concurrente y de constructo.

\section{MÉTODO}

\section{Participantes}

La muestra estuvo integrada por 252 participantes, 243 (96\%) eran mujeres y 9 (4\%) eran hombres. El procedimiento de selección fue no probabilístico por conveniencia. La determinación del tamaño de la muestra atendió al criterio de contar, por lo menos, con diez observaciones por cada ítem a ser analizado de acuerdo con los estándares psicométricos establecidos internacionalmente ${ }^{(39,40)}$.

La media de edad de los cuidadores fue de 37 años con una desviación típica de 8 años. Con respecto a la escolaridad,
191 (76\%) de los participantes contaban con primaria, 38 (15\%) con secundaria, $4(2 \%)$ con preparatoria y 19 (7\%) con licenciatura. La distribución de los participantes respecto a la ocupación mostró que eran: $163(65 \%)$ amas de casa, 49 (19\%) empleados, 18 (7\%) comerciantes, 12 (5\%) profesionistas, 7 (3\%) desempleados y 3 (1\%) estudiantes.

Por lo que respecta al estado civil fueron: 123 (49\%) casados, 68 (27\%) vivían en unión libre, 37 (15\%) solteros, 19 (7\%) divorciados y 5 (2\%) viudos. Y respecto al parentesco entre el cuidador y la persona a la que cuida 232 (92\%) eran las madres, $10(4 \%)$ eran los padres y $10(4 \%)$ eran otros familiares.

Las personas a las que cuidan se distribuyeron de la siguiente forma: respecto al género eran 127 (51\%) niñas y 125 (49\%) niños. La edad osciló entre 3 meses a 18 años, con una media de 9 años y una desviación de 5 . Y respecto al diagnóstico 90 (36\%) tenían Cáncer, 85 (34\%) Diabetes Mellitus y 77 (31\%) Epilepsia.

\section{Instrumentos}

1. Formato de consentimiento informado (revisado y autorizado por el Comité de Ética de la institución).

2. Hoja de datos sociodemográficos. Como edad, género, ocupación, escolaridad, etc.

3. Escala de carga del cuidador de Zarit (Caregiver burden interview, CBI) (11). Adaptada al castellano por Montorio et al. ${ }^{(8)}$, en cuidadores de personas mayores dependientes. Es un instrumento de autoinforme que evalúa la carga de los cuidadores primarios a partir de identificar los sentimientos habituales que experimentan quienes cuidan a otra persona. Está compuesta por 22 ítems tipo Likert con cinco niveles de respuesta: $1=$ nunca, $2=$ rara vez, $3=$ algunas veces, $4=$ bastantes veces $y$ $5=$ siempre. Explica el 55\% de la varianza 
y se distribuyen en tres factores: 1$)$ impacto del cuidado, 2) relación interpersonal y 3) expectativas de autoeficacia. Tiene una consistencia interna que oscila entre .69 y. 90.

4. Cuestionario General de Salud (General Health Questionnaire, GHQ-28) ${ }^{(41)}$. Adaptado en México por Romero-Mendoza y Medina-Mora ${ }^{(42)}$ en estudiantes universitarios. El instrumento detecta casos potenciales de trastornos psiquiátricos de menos de dos semanas de duración. Consta de 28 ítems tipo Likert con cuatro niveles de respuesta (no, para nada; no más de lo habitual; bastante más que lo habitual y mucho más que lo habitual) que son codificadas de 0 a 3 . Está compuesto por cuatro factores: ideación suicida, integrada por los reactivos 11, 20, 22, 24, 25, 27 y 28; depresión leve, compuesta por los ítems 5, 12, 10, 13, 14, 15 y 18; depresión severa, integrada por los reactivos $1,2,7$, $8,9,17$ y 21 ; y ansiedad, compuesta por los ítems 3, 4, 6, 16, 19, 23 y 26. Estos factores explican el $39 \%$ de la varianza y mostraron alfas que van de 0,75 a 0,80.

Este instrumento se utilizó en el presente estudio como criterio para obtener la validez concurrente de la escala de carga de Zarit, ya que se ha observado que existe una relación entre la aparición de síntomas psiquiátricos y la percepción de carga en el cuidador ${ }^{(8,10,12,13)}$.

5. Cuestionario de estrés percibido (Perceived Stress Scale, PSS) ${ }^{433}$. Adaptado en México por Zamora-Macorra ${ }^{(53)}$ en estudiantes de bachillerato. Es un instrumento que evalúa el nivel de control de estrés percibido. Está integrado por 10 ítems tipo Likert con cinco niveles de respuesta (nunca, casi nunca, algunas veces, frecuentemente y siempre) que son codificados en una escala de 0 a 4 , en unifactorial y tiene un alfa de Cronbach de 0,82.

Esta escala se utilizó para confirmar la validez de constructo contrastándola contra los puntajes de la escala de carga del cuidador de Zarit. Ya que se ha observado en otros estudios una relación directamente proporcional entre el estrés y la percepción de carga del cuidador primario informal(13).

\section{Procedimiento}

La escala de carga del cuidador de Zarit realizada por Montorio et al. ${ }^{(8)}$, se sometió a revisión por 4 jueces, expertos en el tema (Psicólogos de la salud o Psicooncólogos), quienes revisaron la coherencia gramatical de la escala. Hubo consenso entre los jueces respecto a que se modificaron las opciones de respuesta de nunca, rara vez, algunas veces, bastantes veces y siempre a nunca, casi nunca, algunas veces, frecuentemente y siempre, para que fueran más claras y se adecuaran al español que se habla en México, tanto en sintaxis como en gramática.

La versión resultante se aplicó a 15 cuidadores primarios informales de niños para evaluar la comprensión y claridad de la prueba. No fue necesario realizar modificaciones adicionales porque el instrumento fue claro para los participantes.

Después, se integró una batería con los instrumentos mencionados en el apartado correspondiente y un psicólogo especialista entrenado en la atención de cuidadores llevó a cabo la aplicación en las instalaciones de un hospital público a lo largo de siete meses. En todos los casos se les invitó a participar, se les explicó el propósito del estudio y se les pidió que firmaran el consentimiento informado. Cada participante contestó de manera individual los cuestionarios en una sesión de aproximadamente 30 minutos. La batería siempre se aplicó en el mismo orden: consentimiento informado, hoja de datos sociodemográficos y médicos, escala de carga del cuidador de Zarit, cuestionario general de salud y cuestionario de estrés percibido. 
En caso de que hubiera alguna duda se orientó a los participantes de tal forma que pudieran contestar los instrumentos cuidando no sesgar las respuestas. Cabe señalar que durante la aplicación de la batería cuatro participantes presentaron crisis y en consecuencia el aplicador les dio contención y los canalizó a un terapeuta de la institución siguiendo las reglas éticas. Finalmente, al terminar la aplicación se les solicitó un correo electrónico o teléfono para hacerles saber los resultados de sus pruebas.

\section{Diseño}

De acuerdo con la clasificación sugerida por Montero y León ${ }^{(44)}$, se trata de un estudio instrumental.

\section{Análisis de datos}

Para valorar las propiedades psicométricas de la escala de carga del cuidador de Zarit, se realizó un análisis de discriminación de reactivos mediante la prueba $t$ de Student, un análisis factorial exploratorio y un análisis factorial confirmatorio para determinar la estructura conceptual de la escala, y finalmente un análisis de las relaciones entre la escala de carga con la de salud y la de estrés percibido mediante el coeficiente de correlación de Pearson para obtener evidencias de su validez. Asimismo, se calculó el coeficiente alfa de Cronbach para determinar su confiabilidad.

\section{RESULTADOS}

Con el fin de identificar la capacidad discriminativa de cada uno de los reactivos se realizó una prueba $t$ de Student para muestras independientes entre el grupo con las calificaciones más altas y el grupo con las calificaciones más bajas, tomando como criterio de clasificación los percentiles 75 y 25, respectivamente. Los 22 ítems mostraron un poder discriminativo pues todos los valores $t$ fueron significativos al nivel de 0,01.

Posteriormente, se realizó un análisis factorial exploratorio utilizando el método de máxima verosimilitud con rotación ortogonal varimax. El índice Kaiser-MeyerOlkin mostró un valor de 0,86. El test de Esfericidad de Bartlett fue significativo Chi cuadrada $=1040,27, p=0,000$, indicativo de la presencia de correlaciones significativas y la pertinencia de llevar a cabo el análisis factorial. El criterio que se siguió para considerar un reactivo dentro de un factor fue que presentara una carga factorial igual o mayor a 0,40 y sin carga factorial similar en otro factor. Una vez considerados estos criterios se eliminaron siete ítems quedando la escala conformada por tres factores que explicaron el 50\% de la varianza.

El primer factor denominado impacto del cuidado quedó integrado por los reactivos 2, 3, 10, 11 y 12 . Hace referencia a aquellas cuestiones relacionadas con los efectos que la prestación de cuidados a un familiar tiene para el cuidador.

El segundo factor Ilamado relación interpersonal quedó integrado por los reactivos 5, 6, 9, 13 y 19. Hace referencia a aquellas emociones o sentimientos que aparecen en la relación que el cuidador mantiene con la persona a la que cuida: agobio, vergüenza, enfado, tensión, incomodidad e indecisión hacia su familiar y su cuidado. $Y$ finalmente el tercer factor denominado expectativas de autoeficacia (este último término entendido de acuerdo con la clasificación de Bandura, 1987), quedó integrado por los reactivos 20 y 21. Reflejan las creencias del cuidador sobre su capacidad para cuidar a su familiar. Tal como se observa en la tabla 1.

El análisis de consistencia interna se realizó mediante el coeficiente alfa de Cronbach para determinar la confiabilidad de la escala total y de cada una de las sub- 
escalas resultantes. La escala total tuvo un índice alfa=0,84. Los factores y sus valores de consistencia interna se pueden consultar en la tabla 1.

Posteriormente, a fin de probar la estructura factorial obtenida, se llevó a cabo un análisis factorial confirmatorio mediante la técnica de ecuaciones estructurales.

Los resultados mostraron que el modelo propuesto tuvo un buen ajuste como lo indican los diferentes índices de ajuste: $X^{2}=71 \quad g l=51, \quad p=0,034, \quad$ RMSEA $=$ 0,04, $\mathrm{GFI}=0,95, \mathrm{AGFI}=0,93, \mathrm{NFI}=0,93$, $\mathrm{CFI}=0,98, \mathrm{IFI}=9,98, \mathrm{TLI}=0,97$. Cabe acla- rar que el índice $X^{2}$ fue significativo, lo que señalaría un mal ajuste, pero este índice es sensible al tamaño muestral, volviéndose significativo cuando las muestras son mayores a 200, siendo éste el caso en este estudio. Por ello, se presentan adicionalmente, los otros índices para valorar el ajuste del modelo. Para dichos índices valores iguales o superiores a 0,90 señalan un buen ajuste, a excepción del CFI que debe ser 0,95 y RMSEA, cuyo valor debe ser menor a 0,05, tales criterios se cumplen cabalmente en los índices reportados. En la figura 1 se presenta el modelo.

\section{Tabla 1. Estructura factorial de la escala de carga del cuidador de Zarit.}

Factor 1. Impacto del cuidado, Factor 2. Relación interpersonal y Factor 3. Expectativas de autoeficacia

\begin{tabular}{|c|c|c|c|}
\hline \multirow{2}{*}{ Reactivos } & \multicolumn{3}{|c|}{ Factores } \\
\hline & 1 & 2 & 3 \\
\hline 11. Falta de intimidad & 0,684 & & \\
\hline 12. Vida social afectada negativamente & 0,676 & & \\
\hline 3. Agobio por compatibilizar responsabilidades & 0,628 & & \\
\hline 2. Insuficiente tiempo propio & 0,603 & & \\
\hline 10. Deterioro de la salud & 0,499 & & \\
\hline 9. Se siente tenso cerca de la persona a la que cuida & & 0,705 & \\
\hline 19. Se siente indeciso sobre la persona a la que cuida & & 0,571 & \\
\hline 6. Vida familiar afectada negativamente & & 0,531 & \\
\hline 13. Distanciamiento de las amistades & & 0,492 & \\
\hline 5. Se siente enfadado cerca de la persona a la que cuida & & 0,487 & \\
\hline 20. Debería hacer más por la persona a la que cuida & & & 0,996 \\
\hline 21. Podría cuidar mejor a la persona a la que cuida & & & 0,668 \\
\hline Valor propio (valor eigen) & 2,48 & 2,02 & 1,49 \\
\hline Media & 10,82 & 8,86 & 7,53 \\
\hline Desviación típica & 4,49 & 3,53 & 2,34 \\
\hline Varianza explicada & 20,69 & 16,88 & 12,48 \\
\hline Alfa de Cronbach & 0,81 & 0,75 & 0,80 \\
\hline
\end{tabular}


Figura 1. Modelo de la estructura factorial de la escala de carga del cuidador de Zarit

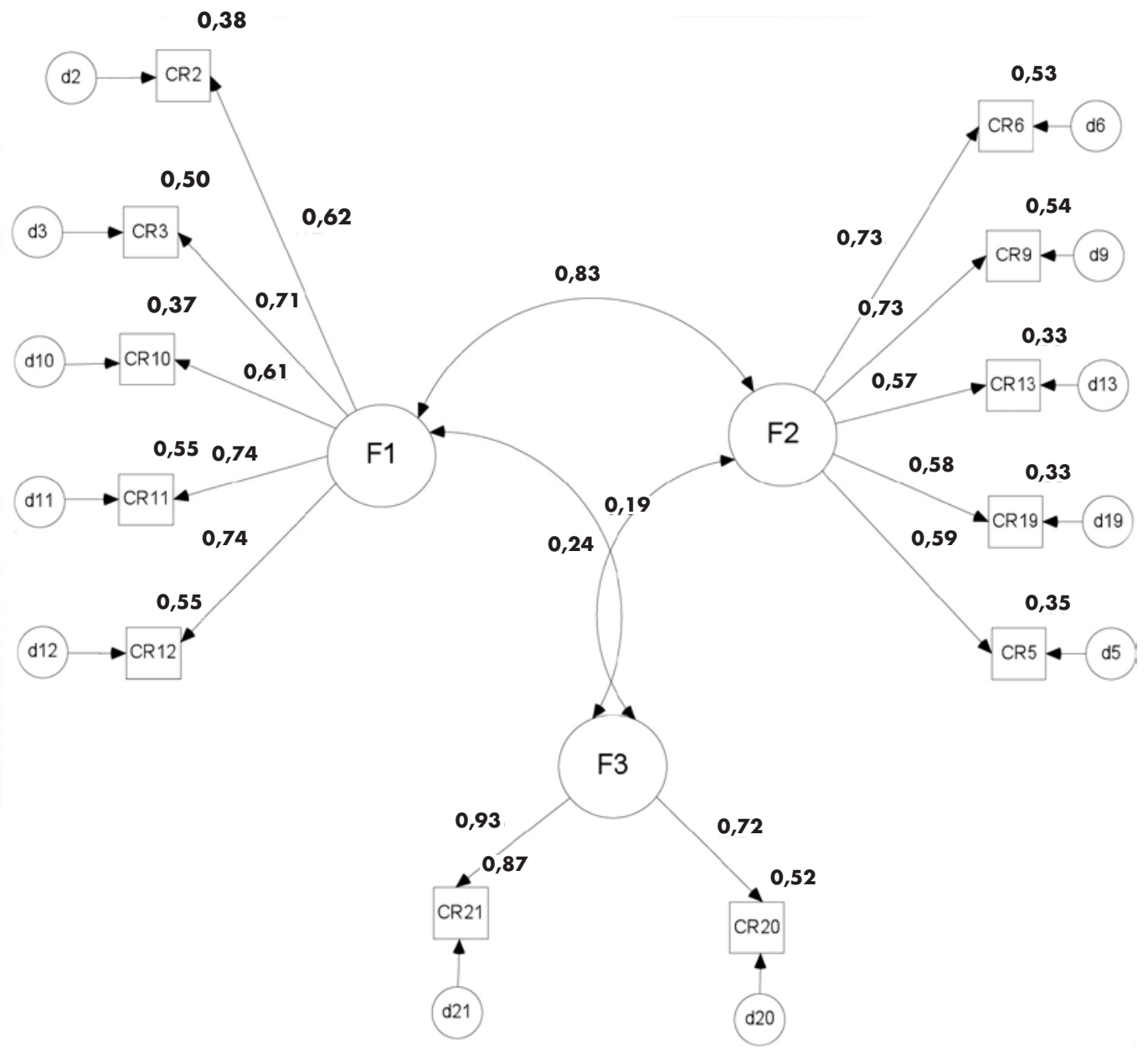

Finalmente, para determinar la validez de criterio concurrente del instrumento se realizó una correlación de Pearson entre las puntuaciones totales obtenidas por los participantes en la CBI y en el GHQ-28, se obtuvo una $r=0,46$, $p=0,000$. La correlación entre los diferentes factores de ambas escalas se muestra en la tabla 2.

Para obtener otra medida sobre la validez de constructo del instrumento se realizó una correlación de Pearson entre las puntuaciones totales obtenidas por los participantes en la CBI y el PSS, se obtuvo una $r=0,36, p=0,000$. La correlación entre los diferentes factores de ambas escalas se muestra en la tabla 3.

\section{DISCUSIÓN}

Se cumplió el propósito del presente estudio al obtener evidencia de validez de 


\section{Tabla 2. Correlaciones de la escala de carga del cuidador de Zarit y el cuestionario general de salud}

\begin{tabular}{|l|c|c|c|c|}
\cline { 2 - 4 } \multicolumn{1}{c|}{} & $\begin{array}{c}\text { Impacto del } \\
\text { cuidado }\end{array}$ & $\begin{array}{c}\text { Relación } \\
\text { interpersonal }\end{array}$ & $\begin{array}{c}\text { Expectativas de } \\
\text { autoeficacia }\end{array}$ & $\begin{array}{c}\text { Carga } \\
\text { global }\end{array}$ \\
\hline Ideación suicida & $0,38^{* *}$ & $0,22^{* *}$ & $0,16^{*}$ & $0,36^{* *}$ \\
\hline Depresión leve & $-0,10$ & $-0,13$ & 0,00 & $-0,11$ \\
\hline Depresión severa & $0,53^{* *}$ & $0,35^{* *}$ & $0,25^{* *}$ & $0,52^{* *}$ \\
\hline Ansiedad & $0,43^{* *}$ & $0,29^{* *}$ & $0,19^{* *}$ & $0,42^{* *}$ \\
\hline Estado general de salud & $0,48^{* *}$ & $0,30^{* *}$ & $0,23^{* *}$ & $0,46^{* *}$ \\
\hline
\end{tabular}

** $\mathrm{p}<0,01$

$* p<0,05$

\section{Tabla 3. Relación de la escala de carga del cuidador de Zarit y el cuestionario de estrés percibido}

Estrés percibido

$\begin{array}{lc}\text { Impacto del cuidado } & 0,38^{* *} \\ \text { Relación interpersonal } & 0,30^{* *} \\ \text { Expectativas de autoeficacia } & 0,09 \\ \text { Carga global } & 0,36^{* *}\end{array}$

** $\mathrm{p}<0,01$

la escala de carga del cuidador de Zarit en una muestra de cuidadores primarios informales de niños con alguna enfermedad crónico degenerativa.

La estructura factorial obtenida en este estudio implicó la eliminación de diez reactivos $(1,4,7,8,14,15,16,17,18$ y 22). La escala final quedó compuesta por 12 ítems que se agruparon en tres factores: impacto del cuidado, relación interpersonal y expectativa de autoeficacia.

Sin embargo, los resultados concuerdan con hallazgos nacionales e internacionales referentes a que la estructura factorial quedó integrada por tres factores ${ }^{(8,10,13,15)}$ y difiere con otros estudios en donde se han encontrado cuatro ${ }^{(14,17)}$ o cinco factores ${ }^{(16)}$.

El factor 1 denominado impacto del cuidado fue el que mostró la mayor cantidad de varianza lo cual coincide con lo reportado tanto en validaciones realizadas con

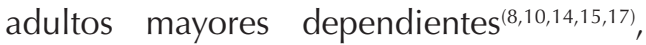
como en adultos con enfermedades crónicas $^{(13)}$. Este factor quedó integrado por los ítems 2, 3, 10, 11 y 12 los cuales aparecen de manera consistente en lo informado en la literatura ${ }^{(8,10,13,15)}$.

Alpuche et al. ${ }^{(13)}$; Martín et al. ${ }^{(10)}$ y Montorio et al. ${ }^{(8)}$, han encontrado en sus estudios, con algunas variaciones, que los 
reactivos 1, 4, 5, 6, 8, 9, 13, 14, 18 y 19 integran el factor 2. En el caso del presente estudio este factor se conformó por los ítems 5, 6, 9, 13 y 19.

El factor 3 se integró con los ítems 20 y 21. Tal como señala la literatura este factor debería contener más ítems ${ }^{(39,40)}$. No obstante, teóricamente es relevante que sólo dos reactivos alcancen un alfa de 0,80. Sin embargo, indudablemente deberán realizarse las revisiones teóricas pertinentes que lleven a la incorporación de más reactivos.

Finalmente, en el factor 3 relacionado con las expectativas de autoeficacia sólo se mantuvieron los ítems que hacen referencia a que los participantes "deberían hacer más por la persona a la que cuido" y a que "podrían cuidarla mejor". Esto se justifica con lo encontrado por otros investigadores $^{(23,35,45,46,54)}$, respecto a que los sentimientos del cuidador hacia la persona a la que cuida tienen una gran influencia en el nivel de sobrecarga cuando el cuidador tiene la creencia de que no cuidó de la mejor manera posible a su hijo y por eso enfermó; por ello, ahora tienen que atender mejor a la persona que cuida ${ }^{34,47)}$. Esta percepción de autoeficacia baja podría estar asociada con las expectativas que tenga sobre la recuperación de la persona a la que cuida; sin embargo, en ocasiones estas resultan incongruentes con el diagnóstico y el pronóstico ${ }^{(48,49)}$.

Las diferencias entre la estructura factorial de la escala original y la que se reporta en el presente artículo posiblemente se deban a la edad y a las manifestaciones clínicas de la enfermedad crónica que tienen los receptores de cuidado, ya que en la versión original se trataba de adultos mayores con algún tipo de demencia y en esta de niños con enfermedades crónicas.

Una posible explicación de porqué los diez reactivos se excluyeron de la estructura factorial de la escala original es que estos reactivos no discriminan entre los grupos de puntajes bajos y altos de carga como ha ocurrido en lo reportado por diversos estudios con cuidadores de adultos con demencia ${ }^{(8,10,14,15,17)}$; es decir, hubo un conceso en las respuestas de la mayoría de los participantes; esto es, se sesgaron hacia un polo de las opciones: "siempre o nunca" esto debido a las características de la muestra que se utilizó en el estudio.

Por ejemplo en los reactivos 7, 8 y 15 la mayoría de los participantes respondieron que "siempre tienen miedo por el futuro de la persona a la que cuidan", esto ocurre posiblemente porque perciben que la vida del niño está siendo amenazada por la enfermedad crónica que tiene y esto atenta el ciclo "natural" de la vida donde se supone que los seres vivos nacen, crecen, se reproducen y mueren; sin embargo, este curso se altera con la aparición de la enfermedad generando impotencia y enojo en las madres porque nunca esperan que un niño pueda tener una enfermedad que ponga en riesgo su vida, como en el caso de los adultos mayores donde esta situación sería "natural y hasta esperable"(50).

Y "siempre consideran que no tienen suficiente dinero para atender a la persona a la que cuidan y además cubrir los otros gastos" la tendencia observada en este ítems posiblemente resulto de esta forma porque la muestra proviene de una nivel socioeconómico de subsistencia; es decir, su nivel de ingresos sólo les permite tener acceso a lo mínimo para vivir.

Mientras que en los reactivos 1, 4, 18 y 22 la mayoría de los participantes consideraron que la persona a la que cuidan "nunca pide más ayuda de la necesaria y nunca se sienten avergonzados por la conducta de esta" esto ocurre porque para ellas es "normal" que el niño demande atención y haga conductas extrañas que son justificadas por su edad y como efecto de la enfermedad ${ }^{(31,32)}$, mientras que los cuida- 
dores de adultos perciben estas conducta como una forma de llamar su atención y son inaceptables porque los receptores de cuidados aunque están enfermos deberían comportase como adultos ${ }^{(29)}$. De hecho, se ha observado que las conductas "extrañas" que realizan los adultos mayores con demencia y la necesidad de una demanda constante de vigilancia y atención por parte del cuidador aumenta la sobrecarga en este $^{(51)}$.

De igual forma, en los ítems relacionados con la responsabilidad del cuidado $(8,16$ y 17) la mayoría respondieron que "siempre creen que la persona a quien cuidan depende totalmente de ellos" y que "nunca dejarían el cuidado del hijo a otra persona" porque piensan que el cuidado de este es su responsabilidad porque son las madres y por ello son capaces de cuidarlo durante todo el tratamiento de la enfermedad, esto se basa en la creencia antes descrita de que es su "deber" y que nadie puede hacerlo mejor que ella que es su madre a pesar de las consecuencias que esto tenga en diversas áreas de su vida(20,37); mientras que en el caso de los cuidadores de adultos con demencia, influenciados por el impacto del rol en diversas áreas de su vida, pueden experimentar episodios de fatiga en los que se cuestionan la continuidad del cuidado y aparecen sentimientos ambivalentes respecto a ser remplazados por otros miembros de la familia ${ }^{(30)}$.

Finalmente, en los ítems que se refieren explícitamente a estar sobrecargados por la situación de cuidado la mayoría de los participantes respondieron que "nunca"; es decir, no perciben haber perdido el control de su vida ni estar sobrecargadas por el cuidado de su hijo, esto probablemente suceda porque no están pensando en lo que a ellas les ocurre o en cómo se sienten, sino en que su hijo este "bien", la prioridad en ese momento no es su bienestar sino el del hijo ${ }^{(34)}$.
Ante esto, no perciben la sobrecarga ocasionada por el desempeño de su rol porque este es visto como una extensión de sus deberes domésticos, los cuales son percibidos como parte de su identidad de género $^{(38)}$, y en este contexto la sobrecarga que experimenta el cuidador es principalmente subjetiva ${ }^{(8)}$ ocasionada por la impotencia de no poder evitar las consecuencias negativas que la persona que cuidan tiene como parte del tratamiento de su enfermedad y porque a pesar de los constantes esfuerzos que hace no logra que este recupere su salud ${ }^{(52)}$.

Por lo anterior, sería altamente recomendable que estudios futuros se enfoquen a modificar la redacción de estos ítems de forma tal que representen mejor el constructo en esta población.

Una de las principales aportaciones de esta investigación es que está entre las primeras de este tema que se realizan en México, por lo que no se encontraron estudios previos sobre instrumentos que permitan evaluar la carga en cuidadores de niños con enfermedades crónicas; los datos estadísticos permiten concluir que esta escala posee propiedades psicométricas adecuadas para ser utilizada para evaluar el nivel de carga en cuidadores de niños que presentan una enfermedad crónica. Sin embargo, es necesario realizar más estudios y análisis factoriales confirmatorios que validen los resultados encontrados hasta el momento.

Además, los retos a superar, asumiendo las limitantes implícitas por el tipo de muestreo del presente estudio, tienen que ver con la posibilidad de mejorar el porcentaje de varianza explicada mediante la restructuración de los ítems y la elaboración de nuevos reactivos a partir de respuestas de las personas a preguntas abiertas u otras técnicas exploratorias que permitan indagar el constructo de carga emocional en esta población. No obstante lo anterior, el presente estudio contribuye 
aportando evidencia en favor de la escala de carga del cuidador de Zarit que a su vez actúa como una contribución inicial para el desarrollo de un instrumento más completo y apropiado para la medición de este constructo en población hispana.

\section{AGRADECIMIENTOS}

La autora agradece al Consejo Nacional de Ciencia y Tecnología (CONACYT) por la beca otorgada para realizar estudios de Doctorado en Psicología. Así mismo, a las autoridades del Instituto Nacional de Pediatría por las facilidades otorgadas para la realización de las fases experimentales de la presente investigación.

\section{REFERENCIAS BIBLIOGRÁFICAS}

1. Ramos R, Figueroa L. El cuidado informal: elemento esencial en el cuidado de la salud. En R. Ramos, editor. Emergencia del cuidado informal como sistema de salud. México: Porrúa, 2008.

2. López A, Blanco D. La importancia de un buen cuidador: guía para cuidadores de personas dependientes. España: Gobierno de La Rioja, 2006.

3. Vásquez B. La importancia del cuidador en los cuidados paliativos. Informe Médico 2006;8:1-4.

4. Kirk K, Fedele D, Wolfe-Christensen C, Phillips T, Mazur T, Mullins L. Parenting characteristics of female caregivers of children affected by chronic endocrine conditions: a comparison between disorders of sex development and type 1 diabetes mellitus. J Pediatr Nurs 2011;26:e29-36. Doi: 10.1016/j.pedn.2010.10.005

5. Ramos R. Emergencia del cuidado informal como sistema de salud. México: Miguel Ángel Porrúa. 2008.

6. Espín A. Caracterización psicosocial de cuidadores informales de adultos mayores con demencia. Rev Cub Salud Pública 2008:34. En línea. [Acceso el 15 de di- ciembre de 2011] Disponible en: http:// scielo.sld.cu/pdf/rcsp/v34n3/spu08308. pdf

7. Lawrence V, Murray J, Samsi K, Banerjee S. Attitudes and support needs of black Caribbean, south Asian and white British carers of people with dementia in the UK. Br J Psychiatry, 2008:193:240-6. Doi:10.1192/bjp.bp.107.045187

8. Montorio C, Izal F, López L, Sánchez C. La entrevista de carga del cuidador. Utilidad y validez del concepto de carga. An Psicol 1998:14:229-48.

9. Grad J, Sainsbury P. Mental illness and the family. Lancet 1963;9:544-7. Doi:10.1016/ S0140-6736(63)91339-4

10. Martín M, Salvadó I, Nadal S, Miji L, Rico M, Lanz P, et al. Adaptación para nuestro medio de la escala de Sobrecarga del cuidador de Zarit. Rev Gerontol 1996;6:33846.

11. Zarit S, Reever K, Bach-Peterson J. Relatives of the impaired elderly: Correlates of feeling of burden. Gerontologist 1980;20;649-55.

12. Zarit $\mathrm{S}$, Orr N, Zarit J. The hidden victims of Alzheimer's disease. Families under stress. New York: University Press. 1985.

13. Alpuche R, Ramos R, Rojas R, Figueroa L. Validez de la entrevista de carga de Zarit en una muestra de cuidadores primarios informales. Psicol Salud 2008; 18;237-45.

14. Da Cruz S. Adaptation and validation of Zarit Burden Interview Scale. Revista Referencia 2010;2:9-16.

15. Martin-Carrasco $M$, Otermin $P$, PérezCamo V, Pujol J, Agüera L, Martin J, et al. EDUCA study: Psychometric properties of the Spanish version of the Zarit Caregiver Burden Scale. Aging Mental Health 2010:14:705-711. Doi:10.1080/13607860903586094.

16. Özlü A, Yildiz M, Aker T. A reliability and validity study on the Zarit caregiver burden scale. Arch Neuropsychiatry 2009:46:38-42. 
17. Yoon E, Robinson M. Psychometric properties of the Korean version of the Zarit Burden Interview (K-ZBI): Preliminary analyses. J Social Work 2005:6:75-86.

18. Mobolaji U, Niran O, Oluyemi C, Mashudat A. Factors associated with caregiver burden in a child and adolescent psychiatric facility in Lagos, Nigeria: A descriptive cross sectional study. BMC Pediatr 2011;12;100-10. Doi:10.1186/14712431-11-110.

19. Pruthi G, Mohta A. Psychosocial burden and quality of life in parents of children with anorectal malformation. J Indian Asso Pediatr Surg 2010;15:15-18. Doi: 10.4103/0971-9261.69135.

20. Rubira E, Munhoz G, Martínez E, Barbosa D, Silva A. Taking care of children with cáncer: Evaluation of the caregiver's burden and quality of life. Rev Latinoam Enfermería 2011;19;515-22.

21. Arredondo T. Calidad de vida y sobrecarga en cuidadores primarios informales de niños con Diabetes Mellitus Tipo 1 y madres de niños sanos. Tesis de Licenciatura no Publicada. México: Facultad de Estudios Superiores Zaragoza/Universidad Nacional Autónoma de México. 2008.

22. Bella G, García M, Spadari-Bratfisch R. Salivary cortisol, stress and health in primary caregivers (mothers) of children with cerebral palsy. Psychoneuroendocrinology 2011;36:834-42.

23. Martínez G, Robles R, Ramos R, Santiesteban M, García V, Morales E, et al. Carga percibida del cuidador primario del paciente con parálisis cerebral infantil severa del Centro de Rehabilitación Infantil teletón. Rev Mex Med Física Rehabilitación 2008;20:23-29.

24. Martínez L. Carga y dependencia percibidas en cuidadores primarios informales de pacientes con parálisis cerebral infantil severa. Tesis de Licenciatura no Publicada. México: Facultad de Estudios Superiores de Zaragoza/Universidad Nacional Autónoma de México. 2010.
25. Martínez L, Ramos R, Robles R, Martínez G, Figueroa L. Carga y dependencia en cuidadores primarios informales de pacientes con parálisis cerebral infantil severa. Psicol Salud 2012;22:275-282.

26. Meltzer L. Mothers of children with chronic illnesses: A caregiver burden model and summer camp as respite care. Sciences Engineering 2003;63:49-15.

27. Pearlin L, Mullan J, Semple S, Skaff M. Caregiving and stress process: an overview of concepts and their measures. Gerontologist1990:30;583-95. Doi: 10.1093/geront/30.5.583

28. Aldana G, Guarino L. Sobrecarga, afrontamiento y salud en cuidadoras de pacientes con demencia tipo Alzheimer. Revista de Psicología de la Universidad Santo Tomás de Chile 2012;9:5-14.

29. Nichols L, Martindale-Adams J, Burns R, Graney M, Zuber J. Translation of a dementia caregiver support program in a health care system.. Arch Intern Med 2011;171:353-9. Doi: 10.1001/archinternmed.2010.548.

30. Rivera M, Dávila M, González P. Calidad de vida de los cuidadores primarios de pacientes geriátricos de la clínica de Medicina Familiar Dr. Ignacio Chávez, ISSSTE. Revista de especialidades Médico-Quirúrgicas 2011;16:27-32.

31. Aung L, Saw S, Chan M, Khaing T, Quah T, Verkooijen $\mathrm{H}$. The hidden impact of childhood cancer on the family: A multi-institutional study from Singapore Ann Acad Med Singapore 2012;41:170-5.

32. Cernvall M, Alaie I, Essen V. The factor structure of traumatic stress in parents of children with cancer: A longitudinal analysis. J Pediatr Psychol. 2012;37::44857. Doi: 10.1093/jpepsy/jsr105

33. Figueroa-Duarte A. Estudio clínico, epidemiológico y sociocultural de la epilepsia. Archivos de Neurociencia 2010;15:139151.

34. Haugstvedt A, Wentzel-Larsen T, Rokne B, Graue M. Perceived family burden 
and emotional distress: similarities and differences between mothers and fathers of children with type 1 diabetes in a population-based study. Pediatr Diabetes 2011:12:107-14. Doi: 10.1111/j.13995448.2010.00661.x

35. Kim K, Lee E, Namkoong K, Lee Y, Lee J, Kim H. Caregiver's burden and quality of life in mitochondrial disease. Pediatr Neurol 2010;42:271-6. Doi: 10.1016/j.pediatrneurol.2009.11.012

36. Yi-Frazier J, Hood K, Case D, Waitzfel$\operatorname{der}$ B, Anderson A, Bloch C, et al. Caregiver reports of provider recommended frequency of blood glucose monitoring and actual testing frequency for youth with type 1 diabetes. Diabetes Res Clin Prac 2012;95:68-75. Doi: 0.1016/j.diabres.2011.08.026

37. Beck A, López M. Tensão devido ao papel de cuidador entre cuidadores de criança com câncer. Rev Bras Enferm 2007; 60:513-8. Doi: 10.1590/S003471672007000500006

38. Young B, Dixon-Woods M, Findlay M, Heney D. Parenting in a crisis: Conceptualising mothers of children with cancer. Soc Sci Med 2002;55:1835-47. Doi: 10.1016/ S0277-9536(01)00318-5

39. Carretero-Dios H, Pérez C. Normas para el desarrollo y revisión de estudios instrumentales. Int J Clin Health Psychol 2005;5:521-51.

40. Carretero-Dios H, Pérez C. Normas para el desarrollo y revisión de estudios instrumentales: consideraciones sobre la selección de test en la investigación psicológica. Int J Clinical Health Psychol 2007;7:863-82.

41. Goldberg D, Hillier V. A scaled version of the General Health Questionnaire. Psychol Med 1979;9:139-45.

42. Romero-Mendoza M, Medina-Mora $M$. Validez de una versión del Cuestionario General de Salud para detectar psicopatología en estudiantes universitarios. Salud Mental 1987;10:90-7.
43. Cohen S, Kamarck T, Mermelstein R. A global measure of perceived stress. J Health Soc Behav 1983;24:385-96.

44. Montero I, León O. A guide for naming research studies in Psychology. Int J Clinical and Health Psychol 2007;7:847-62.

45. Wallander J, Thompson R, Alriksson-Smith A. Psychosocial adjustment of children with chronic physical conditions. En: Roberts MC, editor. Handbook of pediatric psychology. New York: Guildford, 2003. p.141-54

46. Klassen A, Raina P, Reineking S, Dix D, Pritchard S, O'Donnell M. Developing a literature base to understand the caregiving experience of parents of children with cancer: A systematic review of factors related to parental health and well-being. Support Care Cancer 2007;15:807-18. Doi: 10.1007/s00520-007-0243-x

47. Lafaurie M, Barón P, León S, Martínez M, Molina Q, Rojas C. Mothers caring for their children with cancer. Rev Colomb Enferm 2009;5:41-52.

48. Castillo E, Chesla A, Echeverry G, Tascón E, Charry M, Chicangana J. Satisfacción de los cuidadores con la atención de adultos y niños con cáncer. Colomb Med 2005;36:43-9.

49. Coletto M, Camara S. Coping strategies and illness perception in parents of children with chronic diseases. Diversitas: Perspectiva en Psicología 2009;5:97-110.

50. Méndez J. Aspectos psicológicos del niño con cáncer. En R. Rivera, editor. El niño con cáncer. Padecimientos comunes para el médico no especialista. México: Textos Mexicanos, 2007.

51. Mausbach T, Dimsdale E, Ziegler G, Mills J, Ancoli-Israel S, Patterson L, et al. Depressive symptoms predict norepinephrine response to a psychological stressor task in Alzheimer's caregivers. Psychosom Med 2005;67:638-42.

52. Lobst $E$, Alderfer $M$, Jane O, Askins $M$, Fairclough D, Katz E, et al. Problem sol- 
ving and maternal distress at the time of a child's diagnosis of cancer in two-parent versus lone-parent households. J Pediatr Psychol 2009;34:817-21. Doi: 10.1093/ jpepsy/jsn140.

53. Zamora-Macorra M. Migración y estrés como predictores del fracaso escolar en estudiantes de bachillerato. Tesis de Licen- ciatura no publicada. México: Facultad de Estudios Superiores Zaragoza/Universidad Nacional Autónoma de México. 2006.

54. Sullivan-Bolyai S, Deatrick J, Gruppuso P, Tamborlane W, Grey M. Constant vigilance: mothers' work parenting young children with type 1 diabetes. J Pediatr Nurs 2003;18:21-9. 
